\title{
Intraoperative template-molded bone flap reconstruction for patient-specific cranioplasty
}

\author{
Serge Marbacher • Lukas Andereggen • \\ Salome Erhardt • Ali-Reza Fathi • Javier Fandino • \\ Andreas Raabe • Jürgen Beck
}

Received: 28 February 2011 /Revised: 5 September 2011 / Accepted: 20 November 2011 /Published online: 6 March 2012

(C) Springer-Verlag 2012

\begin{abstract}
Cranioplasty is a common neurosurgical procedure. Free-hand molding of polymethyl methacrylate (PMMA) cement into complex three-dimensional shapes is often time-consuming and may result in disappointing cosmetic outcomes. Computer-assisted patient-specific implants address these disadvantages but are associated with long production times and high costs. In this study, we evaluated the clinical, radiological, and cosmetic outcomes of a timesaving and inexpensive intraoperative method to mold custom-made implants for immediate single-stage or delayed cranioplasty. Data were collected from patients in whom cranioplasty became necessary after removal of bone flaps affected by intracranial infection, tumor invasion, or trauma. A PMMA replica was cast between a negative form of the patient's own bone flap and the original bone flap with exactly the same shape, thickness, and dimensions. Clinical and radiological follow-up was performed 2 months post-surgery. Patient satisfaction (Odom criteria) and cosmesis (visual analogue scale for cosmesis) were evaluated 1 to 3 years after cranioplasty. Twenty-seven patients underwent intraoperative template-molded patient-specific cranioplasty with PMMA. The indications for cranioplasty included bone flap infection $(56 \%, n=15)$, calvarian tumor resection $(37 \%, n=10)$, and defect after trauma $(7 \%, n=2)$. The mean duration of the molding procedure was $19 \pm 7 \mathrm{~min}$. Excellent radiological implant alignment was achieved in $94 \%$ of the cases. All $(n=23)$ but one patient rated the cosmetic outcome (mean
\end{abstract}

S. Marbacher $\cdot$ L. Andereggen $\cdot$ A. Raabe $\cdot$ J. Beck Department of Neurosurgery, University Hospital Bern, Bern, Switzerland

S. Marbacher $(\bowtie) \cdot$ S. Erhardt $\cdot$ A.-R. Fathi $\cdot$ J. Fandino Department of Neurosurgery, Kantonsspital Aarau, 3010 Aarau, Switzerland

e-mail: serge.marbacher@ksa.ch
1.4 years after cranioplasty) as excellent $(70 \%, n=16)$ or good $(26 \%, n=6)$. Intraoperative cast-molded reconstructive cranioplasty is a feasible, accurate, fast, and cost-efficient technique that results in excellent cosmetic outcomes, even with large and complex skull defects.

Keywords Patient-specific implant · Cranioplasty · Skull bone flap $\cdot$ Bone replacement material polymethyl methacrylate_PMMA $\cdot$ Brain tumors $\cdot$ Trauma $\cdot$ Brain

\section{Introduction}

Removal of the bone flap is a frequently performed neurosurgical procedure for malignant brain swelling, complex skull fractures, intraosseous tumor invasion, primary or secondary intracranial infections, and intraoperative contamination $[18,37]$. Reimplantation of the patient's own bone flap is the method of choice to restore brain protection and cosmesis as well as to prevent intracranial low pressure syndromes and maintain immunological compatibility [5, 31]. However, primary bone graft reimplantation is often impossible in the case of extensive brain swelling, comminuted fractures, skull bone infection, or tumor infiltration. Additionally, difficulties in storage and timing of surgery may lead to the loss of the bone flap.

Numerous synthetic materials, such as titanium [32], polymethyl methacrylate (PMMA) [13, 28], carbon [41], polyetheretherketone (PEEK) [2], hydroxyapatite (HA) [42], ceramics [27], and osteoconductive bioresorbable materials [8], have been applied to correct skull defects after permanent removal of the bone flap. PMMA is the most frequently used material to restore normal skull contour and shape [28, 37]. It is stable, shows high biocompatibility, and has biomechanical properties comparable to those of cranial 
bone [12]. Whereas smaller defects can be reconstructed without difficulty, reconstruction of larger bone flaps, especially in the forehead and fronto-temporal region, poses a problem [7]. In these cases, free-hand molding of PMMA into complex three-dimensional shapes with correct and uniform thickness is a time-consuming process that can result in unsatisfactory cosmetic outcomes $[11,37]$.

These widely known problems led to the introduction of image-based and computer-assisted manufactured (CAM) implants [42]. Computer-assisted design (CAD) of bone flaps has improved the cosmetic outcome and minimized operation time for adjustment and insertion. However, these techniques are associated with long production times and high costs [42]. In order to avoid the high expenses of CAM/CAD implants, various preoperative cast- and template-based reconstruction methods have been implemented to create similar patient-specific implants (PSI) [1, $4,40]$. Both techniques - CAM/CAD and preoperative template molding - require a second operation. The aim of this study was to evaluate the clinical, radiological, and cosmetic outcomes of a time-saving and inexpensive intraoperative method to mold custom-made implants for either immediate single-stage or delayed cranioplasty.

\section{Materials and methods}

Study patients and procedure-related data

Data were collected from consecutive patients in whom cranioplasty became necessary after removal of bone flaps affected by intracranial infection, tumor invasion, or trauma from June 2006 to June 2009 (Fig. 1). Collected data included sex, age at time of cranioplasty, indication for craniectomy (infection, tumor invasion, or trauma), diagnosis (histology and bacterial culture), localization of craniectomy or cranioplasty, treatment strategy (immediate or delayed surgery), duration of the intraoperative molding procedure and cranioplasty fixation technique (Matrix ${ }^{\mathrm{TM}}$ titanium plates, Craniofix ${ }^{\circledR}$, and non-absorbable sutures), and postoperative adverse events. A wound drain was placed for at least 1 day in all cases. Patients were monitored in the neurosurgical intermediate care unit or the intensive care unit for $24 \mathrm{~h}$ after surgery. Daily clinical observation and routine laboratory checks for infection (leukocytes and Creactive protein), as well as electrolyte controls, until discharge were conducted for all patients.

\section{Molding procedure}

The technique used to create template-molded PSIs has been described previously in a technical note of two patients with cranial defects from infection and tumor invasion, respectively [13]. This molding method has become part of our routine clinical practice. Briefly, the patient's own bone flap served as a template to mold a negative form with the use of PMMA (Palacos ${ }^{\circledR}$ R $2 \times 40$, Essex Chemie AG, Lucerne, Switzerland). After hardening of this negative imprint, the definitive PMMA replica was cast in between the original bone flap and the negative form. This allowed the replica to have exactly the same shape, thickness, and dimensions as the bone flap and negative form (Fig. 2). A fine plastic foil

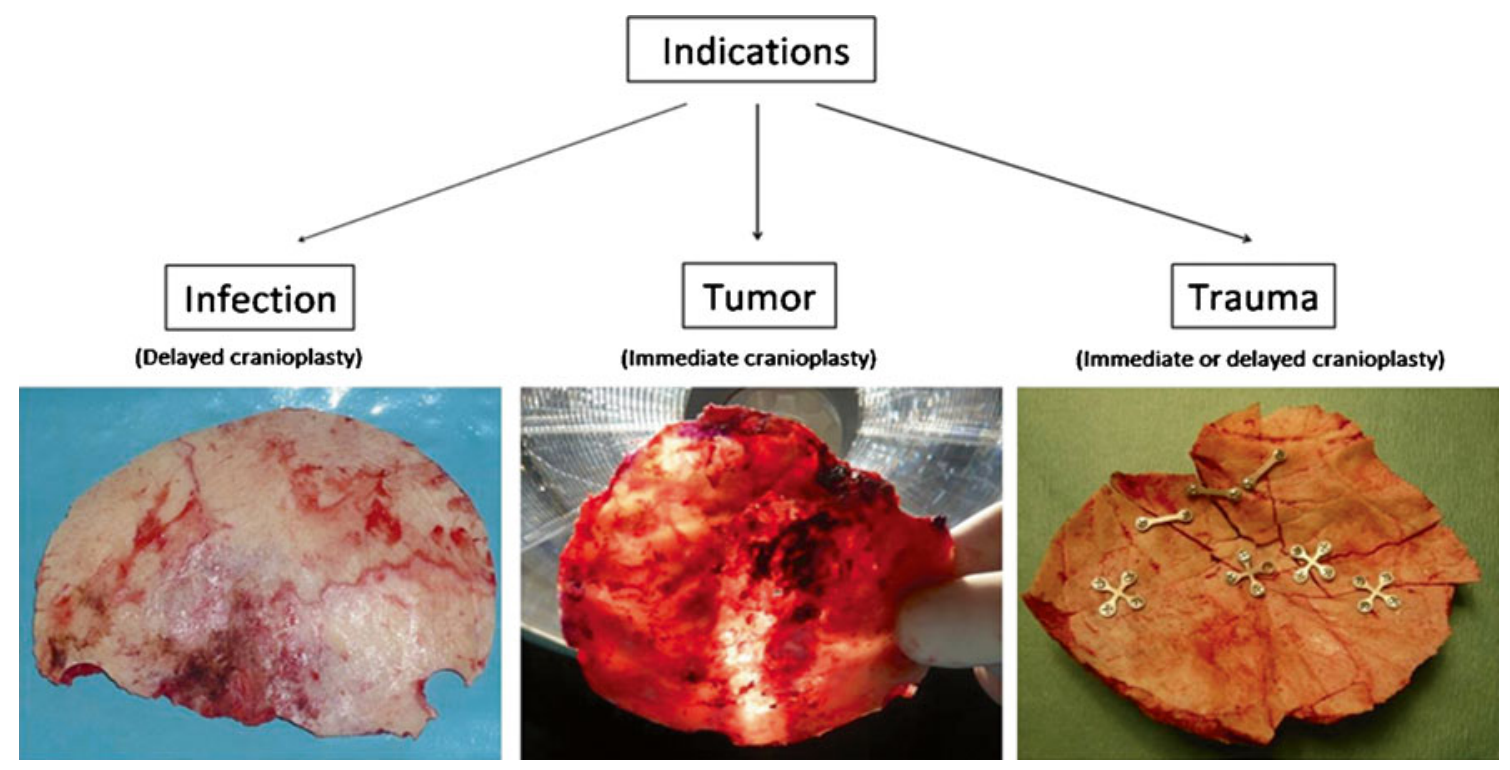

Fig. 1 Intraoperative template-molded bone flap reconstruction for patient-specific cranioplasty can be performed after bone flap loss due to infection, tumor invasion, or comminuted fractures. Whereas restoration of a skull defect due to infection has to be delayed in all cases, tumor- and trauma-associated defects may undergo immediate reconstruction 

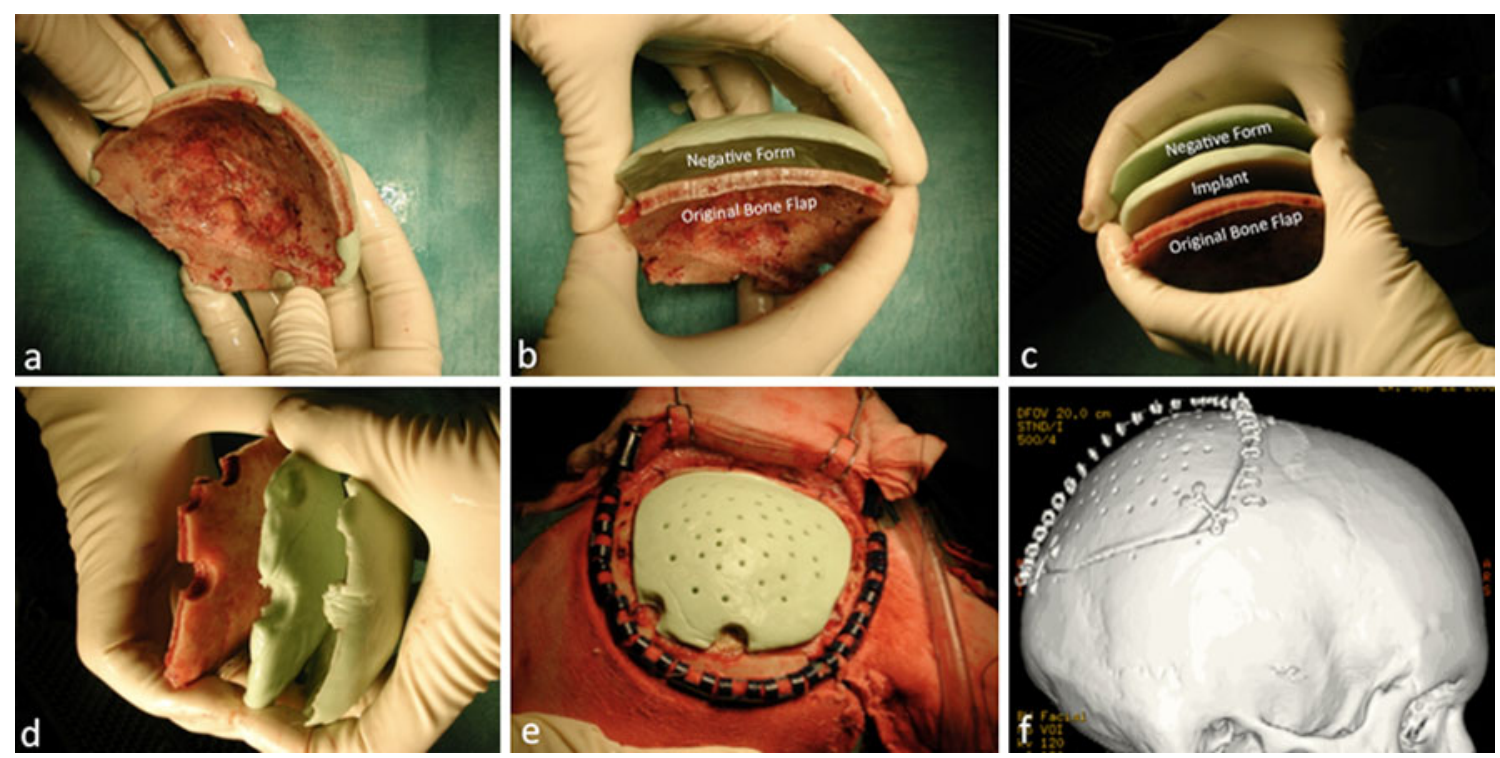

Fig. 2 Palacos ${ }^{\circledR}$ is prepared and molded over the bone flap (a). Excess material is cut along the bone flap edge to obtain an accurate cast (Negative Form, b). After hardening of the negative imprint, the definitive replica is cast in between the bone flap and the negative form (Implant, c). While the Palacos ${ }^{\circledR}$ compound is still in its soft state of aggregation, accurate thickness is achieved through gentle distributed squeezing of the implant in between the negative form and the

or a paraffin oil film was applied in between the bone flap, the replica, and the negative form to prevent adhesion. After sieve-like perforation, the implant was inserted and fixed either with non-absorbable sutures (Ethibond ${ }^{\circledR}$ Excel, Johnson \& Johnson, Ethicon ${ }^{\mathrm{TM}}$, Spreitenbach, Switzerland), the CranioFix ${ }^{\circledR}$ clamp system (B. Braun Medical Inc., Tuttlingen, Germany), or mini-titanium low plates and PlusDrive ${ }^{\circledR}$ cranial screws (Low Profile Neuro Set 1.6; Synthes, Solothurn, Switzerland).

If immediate single-stage reconstruction (e.g., for tumor manifestation, trauma) was not possible (e.g., bone flap infection, contamination), the bone flaps were removed, scrubbed, soaked in antiseptic solution for at least 30 min (Betadine, Mundipharma AG, Basel, Switzerland), and sealed in sterilized biohazard red plastic bags. The unsalvageable bone flaps were sterilized/autoclaved before they served as an intraoperative template $\left(134^{\circ} \mathrm{C}, 18 \mathrm{~min}\right.$, fractionized vacuum, $1,300 \mathrm{mbar}$ [Schaerer AG, Muensingen, Switzerland]). All patients, irrespective of prior infection or not, received a prophylactic single-shot antibiotic treatment with $1.5 \mathrm{~g}$ /day of cefuroxime (IV; Zinacef; Sandoz Pharmaceuticals AG, Steinhausen, Switzerland) $1 \mathrm{~h}$ prior to skin incision. In all cases, it was left to the surgeon's discretion to proceed with a cranioplasty procedure or to postpone cranioplasty due to signs of infection or suspected brain swelling later on in the course of the disease. bone flap. Again, additional Palacos ${ }^{\circledR}$ is cut away, resulting in an implant with exactly the same shape, thickness, and dimensions of the original bone flap (d). Paraffin oil or a fine layer of plastic guarantees an adherence-free molding procedure. After a sieve-like perforation, the implant is reinserted without the need for additional adaptation procedures (e). Accurate alignment is verified in $3 \mathrm{D}$ CT scans (f)

Clinical and radiological endpoints

Clinical and radiological evaluation was conducted during standard follow-up at 2 months post-surgery. The cosmetic appearance on physical examination was graded on a fourtier scale (excellent, good, fair, and poor) based on the surgeon's evaluation and the patient's subjective opinion. Radiological assessment was obtained by computed tomography (CT) scans with three-dimensional (3D) skull reconstruction of the affected site. The studies included a check for signs of infection, hydrocephalus, cerebrospinal fluid (CSF) collections or fistulas, correct alignment of the cranioplasty, and implant size and thickness.

Alignment was considered excellent when the surface dislocation of the implant compared to the skull bone contour was less than $1 \mathrm{~mm}$ (according to $1 \mathrm{~mm}$ section CT images). Alignment was considered accurate if the implant dislocation was at least equal to the thickness of the surrounding skull, and inaccurate if greater than the thickness of the surrounding skull. Thickness of the cranioplasty was compared with the contralateral side in an axial CT scan. Image sections of the greatest diameter of the bone flap in anterior posterior dimension were selected (Fig. 3b). To measure symmetrically, two equal distances of the midline were identified. Then, the same dimensions of an equally measured distance of the bone flap were divided into three identical parts (resulting in three intersection points: \#1-\#3). 

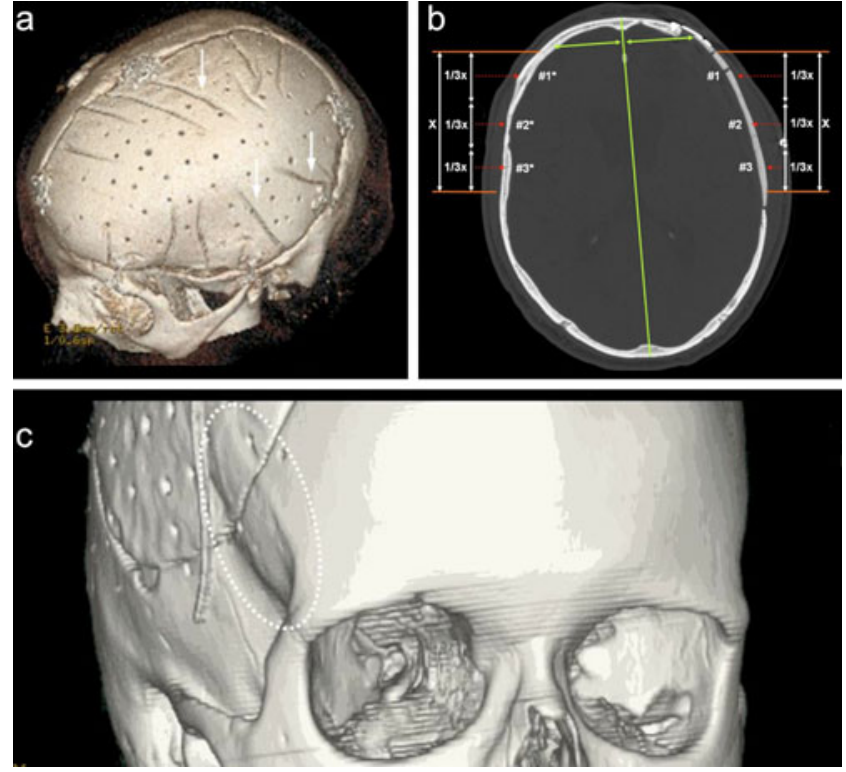

Fig. 3 Infected bone flaps are packed in sterile plastic bags, which cause small wrinkles on the negative imprint and consequently on the final implant (white arrows, a). Due to the accurate molding procedure, the implant thickness does not significant differ when compared to the contralateral mirrored skull (b). With intraoperative template-molded patient-specific implants, exact three-dimensional skull contours can be maintained (especially in complex pterional shapes) (white dashed ellipse, $\mathbf{c})$

In the midline of this third, the skull thickness was measured using automated measurement software (8-slice General Electric Lightspeed Ultra CT scanner, Fairfield, Connecticut, USA). The intersection points (\#1-\#3) were then mirrored on the contralateral skull $(\# 1 * \# 3 *)$ and compared.

Short- and long-term follow-up was conducted after cranioplasty. Patients were evaluated in the outpatient clinic 2 months after surgery and interviewed by telephone 1 to 3 years post-cranioplasty. The evaluation of the telephone interview included the patients' overall satisfaction with the surgical procedure (cranioplasty as a whole) and subjectively experienced cosmesis using the Odom criteria [39] and the visual analogue scale for cosmesis (VASC) [7].

\section{Statistical methods}

Values were expressed as mean \pm standard deviation (SD). Statistically significant differences in procedure-related and outcome data between the indication groups were determined using Student's paired $t$ test. A $p$ value $<0.05$ was considered significant.

\section{Results}

A total of 27 patients underwent intraoperative templatemolded patient-specific cranioplasty (age $54 \pm 19$ years, range 18-78 years). Table 1 includes an overview of patient characteristics and surgical data.

Indications

The indications for cranioplasty were bone flap infection $(56 \%, n=15)$, cranial defect from tumor infiltration $(37 \%$, $n=10)$, and trauma $(7 \%, n=2)$ (Table 1). Bone flap removal

Table 1 Patient characteristics and procedure-related data

\begin{tabular}{|c|c|}
\hline \multicolumn{2}{|l|}{ Patient characteristics } \\
\hline Age $($ mean $\pm \mathrm{SD})$ & $54 \pm 19$ years $(n=27)$ \\
\hline \multicolumn{2}{|l|}{ Sex } \\
\hline Female & $37 \%(n=10)$ \\
\hline Male & $63 \%(n=17)$ \\
\hline \multicolumn{2}{|l|}{ Indication } \\
\hline Infection & $56 \%(n=15)$ \\
\hline Primary & $13 \%(n=2)$ \\
\hline Streptococcus & $100 \%(n=2)$ \\
\hline Secondary & $87 \%(n=13)$ \\
\hline Propionibacterium & $46 \%(n=6)$ \\
\hline Staphylococcus & $23 \%(n=3)$ \\
\hline Streptococcus & $15 \%(n=2)$ \\
\hline Others & $15 \%(n=2)$ \\
\hline Tumor & $37 \%(n=10)$ \\
\hline Meningioma & $70 \%(n=7)$ \\
\hline Hemangiopericytoma & $20 \%(n=2)$ \\
\hline Osteoma & $10 \%(n=1)$ \\
\hline Trauma & $8 \%(n=2)$ \\
\hline \multicolumn{2}{|l|}{ Procedure } \\
\hline $\begin{array}{l}\text { Cranioplasty molding time } \\
(\mathrm{min})^{\mathrm{a}}(\text { mean } \pm \mathrm{SD})\end{array}$ & $19 \pm 7 \min (n=27)$ \\
\hline Infection & $19 \pm 7 \min (n=15)$ \\
\hline Tumor & $20 \pm 6 \min (n=10)$ \\
\hline Trauma (minimum; maximum) & $16 \min (16 ; 16)(n=2)$ \\
\hline Size of cranioplasty ${ }^{b}($ mean \pm SD) & $47 \pm 23 \mathrm{~cm}^{2}(n=27)$ \\
\hline Infection & $57 \pm 18 \mathrm{~cm}^{2}(n=15)$ \\
\hline Tumor & $31 \pm 15 \mathrm{~cm}^{2}(n=10)$ \\
\hline Trauma (minimum; maximum) & $51 \mathrm{~cm}^{2}(63 ; 39)(n=2)$ \\
\hline Single-stage reconstruction (\%) & $39 \%(n=11)$ \\
\hline Infection & $0 \%(n=0)$ \\
\hline Tumor & $35 \%(n=10)$ \\
\hline Trauma & $4 \%(n=1)$ \\
\hline \multicolumn{2}{|l|}{ Fixation technique (\%) } \\
\hline Matrix $^{\mathrm{TM}}$ & $67 \%(n=18)$ \\
\hline Craniofix ${ }^{\circledR}$ & $22 \%(n=6)$ \\
\hline Non-absorbable sutures & $11 \%(n=3)$ \\
\hline
\end{tabular}

${ }^{\mathrm{a}}$ Cranioplasty molding times did not differ significantly $(p=0.33)$ between cases of infection or tumor

${ }^{\mathrm{b}}$ Cranial defects in patients with tumor were not significantly $(p=0.07)$ smaller than those in patients with infection 
due to secondary bone flap infection $(87 \%, n=13)$ was a more frequent procedure than removal for primary infection without previous cranial surgery $(13 \%, n=2)$. In both cases with primary infection, the germ was a Streptococcus. In the case of secondary infection, Propionibacterium acnes was the most common germ $(46 \%, n=6)$, followed by Staphylococcus $(23 \%, n=3)$, and Streptococcus $(n=2)$. In the case of removal of the bone flap due to tumor invasion, immediate cranioplasty was performed in all instances $(n=10)$. Cranioplasty became necessary after surgery for meningiomas in seven patients $(70 \%)$, for hemangiopericytoma in two patients $(20 \%)$, and for osteoma in one patient $(10 \%)$. Instable extensive multi-fragment bone flap fractures were the reason for skull defect repair in two patients $(8 \%)$. Single-stage reconstruction was feasible in one patient without underlying cerebral contusions and no signs of brain swelling. In another patient with extensive skull fracture who suffered significant traumatic brain injury, the fragments were collected, reassembled, and used as template for delayed cranioplasty.

\section{Procedure-related data}

The mean duration of molding the cranioplasty was $19 \pm$ $7 \mathrm{~min}$ ( $n=27$, range $11-34 \mathrm{~min}$ ). Cranioplasty molding times did not differ significantly between cases of infection or tumor (mean $20 \pm 7 \mathrm{~min}, n=15$, range $11-34$ vs $20 \pm$ $6 \mathrm{~min}, n=10$, range $15-27 \mathrm{~min}, p=0.33$ ). Implant sizes ranged from 10 to $106 \mathrm{~cm}^{2}$ (mean size $47 \pm 23 \mathrm{~cm}^{2}$ ). The cranial defects in patients with tumors were not significantly smaller than those in patients with infection (mean $31 \pm$ $15 \mathrm{~cm}^{2}, n=10$, range $17-61$ vs $57 \pm 18 \mathrm{~cm}^{2}, n=15$, range $\left.10-106 \mathrm{~cm}^{2}, p=0.07\right)$. The total manufacturing costs for a single replica amounted to less than 200 United States Dollar (USD), including 90 USD (times two) for the cost of material for the PMMA to mold a negative form and the definitive implant in addition to costs for various expendable items such as biohazard plastic bags, fine plastic foils, and paraffin oil.

Single-stage reconstruction was possible in a substantial number of cases $(39 \%, n=12)$. Immediate cranioplasty was performed in all cases of tumor infiltration $(100 \%, n=10)$ and in one case of trauma with exclusive vast comminuted skull fracture without significant brain contusion and with normal intracranial pressure $(50 \%, n=1$ out of 2$)$. There was no case of immediate cranioplasty in patients $(n=15)$ with loss of their bone flap due to infection.

In most of the cases $(67 \%, n=18)$, the implants were secured using the Craniofix ${ }^{\circledR}$ titanium clamp system with three-point fixation. Matrix ${ }^{\mathrm{TM}}$ titanium plates $(22 \%, n=6)$ and non-absorbable sutures $(11 \%, n=3)$ were less frequently used fixation methods.
Clinical and radiological follow-up

None of the patients showed new postoperative neurological deficits. One patient $(3.7 \%)$ developed fever and meningitis 3 days after surgery. In this case, CT scan confirmed the clinical suspicion of an implant infection, and Klebsiella oxytoca was isolated from the subsequently removed implant. As this patient initially underwent cranioplasty (craniectomy due to severe brain injury/trauma) after bone flap infection caused by another germ (beta-hemolytic Streptococcus group $\mathrm{G}$ ), we assume that there was no relation to our molding procedure. All remaining patients presented with an uneventful postoperative course.

The cosmetic results at 2 months follow-up $(n=26)$ were considered "excellent" in 21 patients $(81 \%)$ and "good" in five patients (19\%) (Table 2). Radiological follow-up at 2 months post-surgery revealed excellent implant alignment in $96 \%$ of cases (Fig. 3a, c). Only one patient (4\%) showed slight overlap of the implant border in relation to the skull bone. In all cases, there were no signs of infection, hydrocephalus, subcutaneous CSF collection, or fistulas. The implant thickness at all three intersection points (\#1-\#3) did not significantly differ when compared to the thickness of the contralateral mirrored skull $(\# 1 *-\# 3 *)(\# 1$ : mean $4.9 \pm 0.3 \mathrm{~mm}$, range $2.8-8 \mathrm{~mm}$ vs $\# 1 *$ : mean $5.1 \pm 0.3 \mathrm{~mm}$, range $2.7-$ $7.8 \mathrm{~mm}, p=0.76$; \#2: mean $4.5 \pm 0.4 \mathrm{~mm}$, range 2.6 $9 \mathrm{~mm}$ vs $\# 2 *$ : mean $4.5 \pm 0.4 \mathrm{~mm}$, range $2.3-9 \mathrm{~mm}$, $p=0.47$; \#3: mean $4.6 \pm 0.4 \mathrm{~mm}$, range $2.5-10 \mathrm{~mm}$ vs \#3*: mean $4.7 \pm 0.5 \mathrm{~mm}$, range $1.8-11 \mathrm{~mm}, p=0.43$ ).

Four patients were lost to long-term follow-up. Three of these patients died unrelated to the cranioplasty procedure and one patient declined to participate in follow-up evaluation. The long-term survey of the remaining patients $(n=23)$ was performed at a mean of $17 \pm 9$ months (range 7-40 months) after cranioplasty. Overall satisfaction with the surgical procedure was considered excellent $(70 \%, n=16)$ or good $(26 \%$, $n=6)$ in all but one patient. The patient with a poor overall outcome $(4 \%, n=1)$ named fluctuating subcutaneous CSF collections as the reason for dissatisfaction. Analysis of the VASC showed that most patients were satisfied with their esthetic outcome (mean $\mathrm{VASC}=8.5 \pm 2.3, n=23$, range $1-$ 10). There were no significant differences in subjectively experienced cosmesis in patients with cranioplasty for infection compared to patients who underwent cranioplasty due to tumor removal (mean $8.9 \pm 1, n=10$, range $7-10$ vs $7.9 \pm 2.5$, $n=11$, range $1-10, p=0.49)$. Clinical outcomes and radiographic findings are summarized in Table 2.

\section{Discussion}

The results of our study demonstrate that intraoperative template-molded PMMA cranioplasty is a fast, safe, precise, 
Table 2 Radiological and clinical outcomes at 2 months and cosmetic results at 1.4 years

\begin{tabular}{|c|c|}
\hline \multicolumn{2}{|c|}{$\begin{array}{l}\text { Results } 2 \text { months post-cranioplasty } \\
(n=26) \\
\text { Clinical follow-up } \\
\text { b }\end{array}$} \\
\hline Excellent & $81 \%(n=21)$ \\
\hline Good & $19 \%(n=5)$ \\
\hline Fair & $0 \%(n=0)$ \\
\hline Poor & $0 \%(n=0)$ \\
\hline \multicolumn{2}{|l|}{ Radiological follow-up ${ }^{a}$} \\
\hline Excellent & $96 \%(n=25)$ \\
\hline Accurate & $4 \%(n=1)$ \\
\hline Inaccurate & $0 \%(n=0)$ \\
\hline \multicolumn{2}{|l|}{ Implant thickness ${ }^{\mathrm{c}}$} \\
\hline Cranioplasty flap (mean $\pm \mathrm{SD}$ ) & $4.7 \pm 1.6 \mathrm{~mm}$ \\
\hline Measurement \#1 & $4.9 \pm 0.3 \mathrm{~mm}$ \\
\hline Measurement \#2 & $4.5 \pm 0.4 \mathrm{~mm}$ \\
\hline Measurement \#3 & $4.6 \pm 0.4$ \\
\hline Mirrored skull (mean \pm SD) & $4.8 \pm 1.8 \mathrm{~mm}$ \\
\hline Measurement $\# 1 *$ & $5.1 \pm 0.3 \mathrm{~mm}$ \\
\hline Measurement \#2* & $4.5 \pm 0.4 \mathrm{~mm}$ \\
\hline Measurement $\# 3 *$ & $4.7 \pm 0.5 \mathrm{~mm}$ \\
\hline \multicolumn{2}{|c|}{$\begin{array}{l}\text { Results } 1.4 \text { years post-cranioplasty } \\
(n=23) \\
\text { Cosmetic results }^{\mathrm{d}}\end{array}$} \\
\hline Patient's VASC $(\text { mean } \pm \mathrm{SD})^{\mathrm{e}}$ & $\begin{array}{l}8.5 \pm 2.3 \text { ( } n=23 \text {, "infection, } \\
\text { tumor, and trauma") }\end{array}$ \\
\hline Infection (mean $\pm \mathrm{SD})$ & $8.9 \pm 1$ ( $n=10$, "infection") \\
\hline Tumor $($ mean \pm SD) & $7.9 \pm 2.5$ ( $n=11$, "tumor") \\
\hline Trauma (mean) (min; max) & $8.7(7.5 ; 9.9)(n=2$, "trauma") \\
\hline \multicolumn{2}{|l|}{ Patient's satisfaction } \\
\hline Excellent & $70 \%(n=16)$ \\
\hline Good & $26 \%(n=6)$ \\
\hline Fair & $0 \%(n=0)$ \\
\hline Poor & $4 \%(n=1)$ \\
\hline
\end{tabular}

${ }^{\mathrm{a}}$ One patient lost for follow-up

${ }^{\mathrm{b}}$ Surgeon's evaluation

${ }^{\mathrm{c}}$ There were no significant differences (\#1 vs \#1*, $p=0.76$; $\# 2$ vs $\# 2 *$, $p=0.47$; \#3 vs \#3*, $p=0.43$ ) in implant cranioplasty thickness when compared to the contralateral mirrored skull

${ }^{\mathrm{d}}$ Four patients lost for follow-up

${ }^{\mathrm{e}}$ Visual analogue scale for cosmesis (VASC) was scored by patients on a scale of $1-10$. There were no significant $(p=0.49)$ differences in subjectively experienced cosmesis in patients with cranioplasty for infection compared to patients who underwent cranioplasty due to tumor removal

and inexpensive technique for reconstructing skull defects after bone flap loss due to infection, tumor invasion, or trauma. Cranioplasty with the presented cast-molded prostheses resulted in favorable cosmesis even in large defects and anatomically complex regions. The intraoperative molding procedure provides immediate single-stage patient- specific cranial reconstruction in a substantial number of patients and spares these patients from potential risks of delayed cranioplasty.

The indication for reconstructive cranioplasty is dependent on the size and location of the defect, but is considered appropriate in defects larger than $10 \mathrm{~cm}^{2}$ in order to protect the subjacent brain against trauma [11]. Any bone defect may eventually lead to depression of the skin and thereby result in a malformed appearance, regardless of size [15]. Psychosocial (stigmatization of mental impairment) and psychological (anxiety stemming from concern about possible trauma) factors may even justify cranioplasty regardless of the defect size $[15,20]$. Along with primary subjective symptoms, including headache, dizziness, fatigability, mental depression, intolerance to vibration, and discomfort around the craniotomy site (especially during warm weather), patients with large skull defects can also develop objective neurological deficits. These symptoms are known as "the syndrome of the trephined" $[19,23]$ and "syndrome of the sinking skin flap", respectively [14, 38, 45].

Although the time up to delayed cranioplasty is only a few months, psychosocial aspects, the feeling of not being protected against trauma, and esthetic considerations during that time should not be underestimated [11]. Prompt cranioplasty spares the patient from above mentioned unpleasant conditions, positively affects cerebral metabolism, and may facilitate patient rehabilitation [30, 44]. Immediate cranioplasty is preferable in all patients and is considered technically no more difficult, if not easier and faster, than delayed procedures [32]. In most patients where the bone flap has to be discarded due to tumor invasion, prompt patient-specific reconstruction using the described method is feasible. In all other cases, it is left to the surgeon's discretion to proceed with the cranioplasty procedure or to postpone cranioplasty due to signs of infection or suspected brain swelling later on in the course of the disease. A single-stage approach to manage skull defects avoids the risk of a second procedure, with potential difficulties caused by soft tissue contracture and scar bridging. Furthermore, costs of a second hospitalization, additional imaging procedures, and surgery can be saved.

Reimplantation of the patient's own bone flap may remain the best procedure to reconstruct cranial defects. In order to salvage infected bone flaps, numerous methods have been proposed, including wash-in, wash-out indwelling antibiotic irrigation systems [3], aggressive surgical debridement and delivery of systemic antibiotics [6], autopurifying by the patient's immune system [46], or autoclavation [21] which has also been advocated in cases of tumor invasion [43]. However, when primary bone graft reimplantation becomes impossible (comminuted fractures, skull bone infection, tumor infiltration, or storage difficulties), cranioplasty using bone substitutes has to be considered. 
Various materials - most frequently PMMA, titanium, and HA - have been used for surgical correction of skull defects. It is unclear which alloplastic graft provides the best overall results, and the choice is mainly based on personal experience and economic considerations. Because of its good biocompatibility, low radiopacity, strong resistance to functional stress, easy handling, and low costs, PMMA is the most frequently used material and is still regarded as the material of choice $[33,37]$. Despite these advantages, one has to be aware of exothermic reactions, toxic fumes during mixing, and rare allergic reactions [26, 29]. Titanium is another widely used implant material because it is fairly radiolucent, neither magnetic nor paramagnetic, and possesses excellent biocompatibility with minimal fibrous encapsulation. Titanium does not cause hypersensitivity reactions, is relatively inexpensive, and shows low graft infection rates compared with PMMA implants, autoclaved grafts, and autogenous grafts [36]. Potential drawbacks for follow-up evaluations (e.g., meningioma) are the suboptimal results of imaging quality due to titanium artifacts [7]. Although much more costly, HA is increasingly used in craniofacial reconstruction. Despite the high biocompatibility (osteoconductivity and osteointegration), inflammatory reactions have been described in various studies, and material costs exceed those of titanium and PMMA [34, 35, 37]. Apart from safety and economical considerations, the primary rationale behind an immediate reconstruction is the potential benefit to functional, esthetic, and psychological outcomes [11, 30, 32].

The costs for CAM/CAD implants greatly exceed the expenses for intraoperative template-molded patientspecific implants. Large ceramic plates can cost from 5,000 to 7,000 USD, whereas titanium implants range from 2,000 to 5,000 USD. The production costs for our intraoperative template-molded PMMA cranioplasty implants averaged 200 USD per piece. Aside from the 10- to 20fold greater manufacturing/production costs, CT workload and radiation exposure can be reduced using the presented technique. Furthermore, in a substantial number of patients, a second operative procedure, with hospitalization and associated costs, can be avoided with an intraoperative singlestage approach.

Correct thickness is required with PMMA cement to create a graft that is uniformly strong, without thin regions and thus without potential fracture sites. Correct positioning and size of the implant not only results in cosmetically favorable outcomes, but also reduces operation time for adjustment and insertion, minimizes bone flap subsidence, wound dehiscence due to tensionless wound closure, wound infection, and finally revision operations [18]. Furthermore, thermal tissue damage caused by an exothermic polymerization reaction during hardening [17] and cytotoxic damage using PMMA implants by direct molding on the lesion [24] have been reported. With the presented procedure, due to the fact that molding is indirect and removed from the involved sensitive tissue, no damage occurs using PMMA. Patientspecific reconstruction for small cranial vault defects, as often seen after surgery for bone invasive tumors or depressed skull fractures, remains controversial. Even though additional costs (100 USD for the negative form) using the described intraoperative template-molded technique are low, operation time is extended by approximately $20 \mathrm{~min}$. In small defects, good cosmetic outcomes and bony alignment can also be achieved by free-hand molding cranioplasty using PMMA or titanium mesh [32].

Infection was the leading indication for alloplastic cranioplasty in this series. The most common germ in our series was $P$. acnes. This anaerobic Gram-positive bacillus, found as a normal component of the bacterial flora of the skin, is a common causative germ for infection after craniotomy [25]. The most common infecting organism is reported to be Staphylococcus aureus [10], which was the second most common bacterium in our series. The incidence of bone flap infection following routine cranioplasty is about 6-12\% [9, $36,37]$. Cranioplasty in patients with a history of previous infection has approximately $10-15 \%$ higher risk of infection [31]. In a 4-year, retrospective cranioplasty study with 131 patients using PMMA prostheses, clinical follow-up for a minimum of 6 months revealed that the infection rate associated with prefabricated PMMA prostheses was lower $(5.8 \%)$ than that for intraoperatively molded PMMA prostheses (13\%) [28]. The infection rate in the study patient population remains to be determined in clinical long-term follow-up. The infection rate of the present series of intraoperatively template-molded PMMA implants (3.7\%) with a clinical follow-up of at least 7 months is comparable to the reported infection rate $(5.8 \%)$ using prefabricated PMMA prostheses [28]. However, long-term infection rate in this study patient population remains to be determined.

Despite all the listed advantages of the presented technique, there is an important limitation. As already noted by Gliese et al. who described a similar method for alloplastic flap modeling, the patient's own bone flap is an irreplaceable element of the described technique [16]. In their elegant technique, a negative imprint of the patient's original bone flap is performed prior to surgery. This cast is then autoclaved and serves as a template at the time of cranioplasty. In our series, all infected bone flaps had to be autoclaved and packed into sterile bags. For extensive comminuted fractures, it was vital to bring together all pieces to achieve a proper template for the molding procedure. In the case of bone resorption or extensive tumor destruction, an adequate template is not available and free-hand techniques or CAM/ $\mathrm{CAD}$ becomes the alternative cranioplasty method. Another option, when the prerequisite of the own bone flap is missing, is to perform split calvarium cranioplasty. Fresh 
autologous bone has the highest possible biocompatibility, fuses with the adjacent bone, and often completely revitalizes. Although none of the patients of the present series were younger than 19 years of age, the split calvarial bone graft technique should be considered for reconstruction of pediatric skull defects [22].

\section{Conclusion}

Intraoperative template-molded cranioplasty using PMMA provides a feasible, fast, and cost-efficient method to precisely reconstruct the patient's own bone flap in terms of alignment and thickness. This molding method is safe, results in excellent cosmetic outcomes, and can be applied in early and delayed cranioplasty procedures. Single-stage reconstruction spares a substantial number of patients from unpleasant conditions with potential benefits for functional, esthetic, and psychological outcomes.

Acknowledgments, funding, and disclosure The authors have no personal, financial, or institutional interest in any of the drugs, materials, or devices described in this article. We are indebted to the radiology technicians from the Institute of Neuroradiology, Bern University Hospital and University of Bern, Bern, Switzerland, for their diligent three-dimensional computer tomography reconstructions. Finally, we are especially grateful for the editorial support of Jeannie Wurz, Department of Intensive Care Medicine, Bern University Hospital, Bern, Switzerland and Susan Wieting, Department of Neurosurgery, Bern University Hospital, Bern, Switzerland.

\section{References}

1. Alesch F, Bauer R (1985) Polyacryl prosthesis for cranioplastytheir production in silicon rubber casts. Acta Neurochir (Wien) 77:68-71

2. Andrzejak S, Fortuniak J, Wrobel-Wisniewska G, Zawirski M (2005) Clinical evaluation of the polypropylene-polyester knit used as a cranioplasty material. Acta Neurochir (Wien) 147:973976

3. Auguste KI, McDermott MW (2006) Salvage of infected craniotomy bone flaps with the wash-in, wash-out indwelling antibiotic irrigation system. Technical note and case series of 12 patients. J Neurosurg 105:640-644

4. Bhargava D, Bartlett P, Russell J, Liddington M, Tyagi A, Chumas P (2009) Construction of titanium cranioplasty plate using craniectomy bone flap as template. Acta Neurochir (Wien) 152(1):173176

5. Bishop A, Gooch R, Eguchi A, Jeffrey S, Smallwood L, Anderson J, Estevez AG (2009) Mitigation of peroxynitrite-mediated nitric oxide (NO) toxicity as a mechanism of induced adaptive NO resistance in the CNS. J Neurochem 109:74-84

6. Bruce JN, Bruce SS (2003) Preservation of bone flaps in patients with postcraniotomy infections. J Neurosurg 98:1203-1207

7. Cabraja M, Klein M, Lehmann TN (2009) Long-term results following titanium cranioplasty of large skull defects. Neurosurg Focus 26:E10

8. Chen TM, Tsai JC, Burnouf T (2008) Cranioplasty using osteoconductive scaffold and platelet glue. J Trauma 65:1321-1327
9. Cheng YK, Weng HH, Yang JT, Lee MH, Wang TC, Chang CN (2008) Factors affecting graft infection after cranioplasty. J Clin Neurosci 15:1115-1119

10. Dashti SR, Baharvahdat H, Spetzler RF, Sauvageau E, Chang SW, Stiefel MF, Park MS, Bambakidis NC (2008) Operative intracranial infection following craniotomy. Neurosurg Focus 24:E10

11. Dujovny M, Aviles A, Agner C, Fernandez P, Charbel FT (1997) Cranioplasty: cosmetic or therapeutic? Surg Neurol 47:238-241

12. Eppley BL (2005) Biomechanical testing of alloplastic PMMA cranioplasty materials. J Craniofac Surg 16:140-143

13. Fathi AR, Marbacher S, Lukes A (2008) Cost-effective patientspecific intraoperative molded cranioplasty. J Craniofac Surg 19:777-781

14. Fodstad H, Love JA, Ekstedt J, Friden H, Liliequist B (1984) Effect of cranioplasty on cerebrospinal fluid hydrodynamics in patients with the syndrome of the trephined. Acta Neurochir (Wien) 70:21-30

15. Gladstone HB, McDermott MW, Cooke DD (1995) Implants for cranioplasty. Otolaryngol Clin North Am 28:381-400

16. Gliese M, Handel G, Brawanski A (2000) A simple and cosmetically ideal method for cranioplasty. Zentralbl Neurochir 61:155-157

17. Golz T, Graham CR, Busch LC, Wulf J, Winder RJ (2010) Temperature elevation during simulated polymethylmethacrylate (PMMA) cranioplasty in a cadaver model. J Clin Neurosci 17(5):617-622

18. Gooch MR, Gin GE, Kenning TJ, German JW (2009) Complications of cranioplasty following decompressive craniectomy: analysis of 62 cases. Neurosurg Focus 26:E9

19. Grant FC, Norcross NC (1939) Repair of cranial defects by cranioplasty. Ann Surg 110:488-512

20. Grantham EC, Landis HP (1948) Cranioplasty and the posttraumatic syndrome. J Neurosurg 5:19-22

21. Jankowitz BT, Kondziolka DS (2006) When the bone flap hits the floor. Neurosurgery 59:585-590

22. Josan VA, Sgouros S, Walsh AR, Dover MS, Nishikawa H, Hockley AD (2005) Cranioplasty in children. Childs Nerv Syst 21(3):200-204

23. Joseph V, Reilly P (2009) Syndrome of the trephined. J Neurosurg 111:650-652

24. Kalteis T, Luring C, Gugler G, Zysk S, Caro W, Handel M, Grifka J (2004) Acute tissue toxicity of PMMA bone cements. Z Orthop Ihre Grenzgeb 142:666-672

25. Kelly ME, Fourney DR, Guzman R, Sadanand V, Griebel RW, Sanche SE (2006) Propionibacterium acnes infections after cranial neurosurgery. Can J Neurol Sci 33:292-295

26. Kirby BS, Doyle A, Gilula LA (2003) Acute bronchospasm due to exposure to polymethylmethacrylate vapors during percutaneous vertebroplasty. AJR Am J Roentgenol 180:543-544

27. Kobayashi S, Hara H, Okudera H, Takemae T, Sugita K (1987) Usefulness of ceramic implants in neurosurgery. Neurosurgery 21:751-755

28. Lee SC, Wu CT, Lee ST, Chen PJ (2009) Cranioplasty using polymethyl methacrylate prostheses. J Clin Neurosci 16:56-63

29. Li C, Mason J, Yakimicki D (2004) Thermal characterization of PMMA-based bone cement curing. J Mater Sci Mater Med 15:85-89

30. Liang W, Xiaofeng Y, Weiguo L, Gang S, Xuesheng Z, Fei C, Gu L (2007) Cranioplasty of large cranial defect at an early stage after decompressive craniectomy performed for severe head trauma. J Craniofac Surg 18:526-532

31. Manson PN, Crawley WA, Hoopes JE (1986) Frontal cranioplasty: risk factors and choice of cranial vault reconstructive material. Plast Reconstr Surg 77:888-904

32. Marbacher S, Andres RH, Fathi AR, Fandino J (2008) Primary reconstruction of open depressed skull fractures with titanium mesh. J Craniofac Surg 19:490-495

33. Marchac D, Greensmith A (2008) Long-term experience with methylmethacrylate cranioplasty in craniofacial surgery. J Plast Reconstr Aesthet Surg 61:744-752 
34. Matic DB, Manson PN (2004) Biomechanical analysis of hydroxyapatite cement cranioplasty. J Craniofac Surg 15:415-422

35. Matic D, Phillips JH (2002) A contraindication for the use of hydroxyapatite cement in the pediatric population. Plast Reconstr Surg 110:1-5

36. Matsuno A, Tanaka H, Iwamuro H, Takanashi S, Miyawaki S, Nakashima M, Nakaguchi H, Nagashima T (2006) Analyses of the factors influencing bone graft infection after delayed cranioplasty. Acta Neurochir (Wien) 148(5):535-540

37. Moreira-Gonzalez A, Jackson IT, Miyawaki T, Barakat K, DiNick V (2003) Clinical outcome in cranioplasty: critical review in longterm follow-up. J Craniofac Surg 14:144-153

38. Nakamura T, Takashima T, Isobe K, Yamaura A (1980) Rapid neurological alteration associated with concave deformity of the skin flap in a craniectomized patient. Case report. Neurol Med Chir (Tokyo) 20:89-93

39. Odom GL, Woodhall B, Wrenn FR (1952) The use of refrigerated autogenous bone flaps for cranioplasty. J Neurosurg 9:606-610

40. Rotaru H, Baciut M, Stan H, Bran S, Chezan H, Iosif A, Tomescu M, Kim SG, Rotaru A, Baciut G (2006) Silicone rubber mould cast polyethylmethacrylate-hydroxyapatite plate used for repairing a large skull defect. J Craniomaxillofac Surg 34:242-246

41. Saringer W, Nobauer-Huhmann I, Knosp E (2002) Cranioplasty with individual carbon fibre reinforced polymere (CFRP) medical grade implants based on CAD/CAM technique. Acta Neurochir (Wien) 144:1193-1203

42. Staffa G, Nataloni A, Compagnone C, Servadei F (2007) Custom made cranioplasty prostheses in porous hydroxy-apatite using $3 \mathrm{D}$ design techniques: 7 years experience in 25 patients. Acta Neurochir (Wien) 149:161-170

43. Vanaclocha V, Saiz-Sapena N, Garcia-Casasola C, De Alava E (1997) Cranioplasty with autogenous autoclaved calvarial bone flap in the cases of tumoural invasion. Acta Neurochir (Wien) 139:970-976

44. Winkler PA, Stummer W, Linke R, Krishnan KG, Tatsch K (2000) The influence of cranioplasty on postural blood flow regulation, cerebrovascular reserve capacity, and cerebral glucose metabolism. Neurosurg Focus 8:e9
45. Yamaura A, Makino H (1977) Neurological deficits in the presence of the sinking skin flap following decompressive craniectomy. Neurol Med Chir (Tokyo) 17:43-53

46. Yano H, Tanaka K, Matsuo T, Tsuda M, Akita S, Hirano A (2006) Cranioplasty with auto-purified bone flap after infection. J Craniofac Surg 17:1076-1079

\section{Comments}

Siamak Asgari, Ingolstadt, Germany

The authors presented a retrospective study about 27 patients, who underwent one- or two-stage surgery for cranioplasty. Indication for cranioplasty were posttraumatic skull defects $(n=2)$, resection of tumors with skull involvement $(n=10)$, and bone flap infection $(n=$ 15). The authors described a method of template-molded PMMA cranioplasty. The authors gave a very detailed analysis of intraoperative characteristics and the postoperative cosmetic results. Altogether, the described cranioplasty procedure is very cost-effective and showed excellent radiological and esthetic results. Finally, the main limitation of this strategy, the autologous bone resorption, was mentioned.

\section{Philippe Bijlenga, Geneva, Switzerland}

With more and more decompressive craniectomies performed for different reasons, a simple, safe, quick, and low-cost solution for cranioplasty, as described in this article, is very welcome.

The authors describe a simple method to generate a negative print of the patient's bone flap that can then be used as a cast to build a Palacos ${ }^{\mathbb{R}}$ cement replica. This article shows that the method is not only feasible but also safe and rapid and presented an excellent radiological and cosmetic outcome in 27 patients. The method that is described in this article presents a long list of advantages and a unique limitation that resides in the absolute need of the original patient's bone flap to serve as a model. Readers are invited to make their own experience and the future will tell us if the technique holds its promises and becomes a worldwide standard. 